\title{
The Prediction of Three Key Properties on Coalbed Methane Reservoir Using Artificial Intelligence
}

\author{
Ahmad F. Hadad ${ }^{1}$, Sudjati Rachmat ${ }^{1}$, Tutuka Ariadji ${ }^{1}$ \&Kuntjoro A. Sidarto ${ }^{2}$ \\ ${ }^{1}$ Petroleum Engineering Department, Institut Teknologi Bandung, Bandung, Indonesia \\ ${ }^{2}$ Mathematics Department, Institut Teknologi Bandung, Bandung, Indonesia \\ Correspondence: Ahmad F. Hadad, Petroleum Engineering Department, Institut Teknologi Bandung, Bandung, \\ Indonesia. E-mail: afhadad76@yahoo.com
}

Received: May 27, 2017

doi:10.5539/mas.v11n8p57
Accepted: June 8, 2017

Online Published: July 10, 2017

\begin{abstract}
This research focuses on creating the prediction tools for the three key properties in coalbed methane (CBM) reservoir; the properties are gas content, Langmuir parameters, and permeability. Basically, their roles are to describe the gas in place and also future dynamic behavior of CBM reservoir. These three key properties are tried to be predicted with open-hole data as the inputs.

It uses artificial neural network (ANN) and adaptive neuro fuzzy inference system (ANFIS) to generate the prediction tools. It is started from data preparation and processing, then pattern or function identifications, and finalized by validation and testing. Several training algorithms are applied for ANN such as adaptive gradient descent (ANN_GDX), Levenberg-Marquardt (ANN_LM), resilient backpropagation (ANN_RP), scaled conjugate gradient (ANN_SCG), and Bayesian regularization algorithm (ANN_BR). The first fives employ the early stopping technique for regularization, and the last one does Bayesian regularization. On the other hand, the ANFIS will use only early stopping technique.

Based on this research, it is concluded that both ANN and ANFIS are able to identify the patterns or function between open-hole log data and the three key properties (TKP). Furthermore, it can be concluded that ANN_LM, ANFIS, and ANN_BR are the best selected algorithms which resulted the lowest error of TKP's predictions.
\end{abstract}

Keywords: coalbed methane, artificial intelligence, prediction tools

\section{Introduction}

Data measurement is the important element of the oil/gas industry business plan. Either in conventional reservoir or unconventional reservoir. It requires significant budget, especially for core analysis and well testing. A good alternative of data measurement and as the most economical way is the application of prediction tools, even though it still have the range of uncertainties. This research will focus on creating the prediction tools for the three key properties (TKP) of coalbed methane (CBM) reservoir.

\subsection{Coalbed Methane}

CBM has different characteristic compared to conventional reservoirs such as sandstone and carbonate. CBM can act both as a source rock and as a reservoir rock. CBM has two porosity systems (dual-porosity); primary porosity and secondary porosity. The primary porosity is the matrix porosity commonly referred to micro-porosity. Secondary porosity or macro-porosity is the network of natural fractures, commonly referred to cleats in CBM terminology.

Tarek Ahmed (2005) in his book stated that the most important data needed to estimate gas in place and other calculations are derived from core analysis. The initial gas in place (IGIP) is the total volume of initial gas that is stored in a coal. It can be calculated using equation 1 .

Where,

$$
\mathrm{G}=1359.7 \mathrm{Ah} \rho_{\mathrm{b}} \mathrm{G}_{\mathrm{c}}
$$

\begin{tabular}{llll}
$\mathrm{G}$ & $=$ Initial gas in place $(\mathrm{scf})$ & $\mathrm{h}$ & $=$ Coal thickness $(\mathrm{ft})$ \\
\hline $\mathrm{A}$ & $=$ Area (acres) & $\rho_{\mathrm{b}}$ & $=$ Bulk density of coal $(\mathrm{gr} / \mathrm{cc})$ \\
\hline & $\mathrm{G}_{\mathrm{c}}$ & $=$ Gas content $(\mathrm{scf} / \mathrm{ton})$
\end{tabular}


The area (A) can be calculated directly from the object (reservoir, sector, or sample), coal thickness (h) and bulk density $\left(\rho_{\mathrm{b}}\right)$ are commonly from open-hole log data, and gas content is from core analysis result.

Gas content is determined by measuring the volume of total gas released and the weight of the sample. It is usually reported in units of scf/ton. Gas content provides the information of gas volume in a coal, but it cannot provide the information of dynamic behavior. Therefore, it requires additional data such as reservoir pressure and the sorption isotherm parameters of the coal to explain dynamic condition that will be happen. This research uses Langmuir isotherm parameters, such as $\mathrm{V}_{\mathrm{L}}$ and $\mathrm{P}_{\mathrm{L}}$.

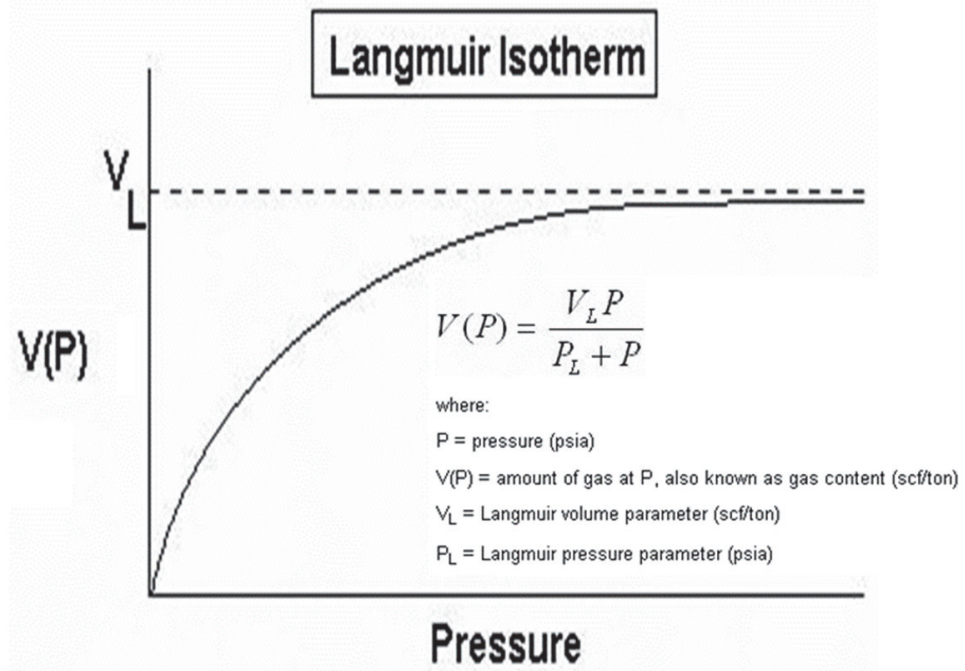

Figure 1. Langmuir Isotherm Curve (Fekete, 2014)

Langmuir volume $\left(\mathrm{V}_{\mathrm{L}}\right)$ is the maximum gas that can be adsorbed on a piece of coal at infinite pressure. Meanwhile, Langmuir pressure $\left(\mathrm{P}_{\mathrm{L}}\right)$ is the pressure at which half of the Langmuir volume can be adsorbed. At reservoir condition, saturated coal, gas content lies inline with Langmuir curve. Meanwhile, for under-saturated coal, gas content lies below the Langmuir curve. Furthermore, the recovery factor of gas in sorption scale will follow the Langmuir curve until abandonment pressure. Figure 1 shows the Langmuir isotherm curve.

It is common that the gas from CBM wells is dominated by methane $\left(\mathrm{CH}_{4}\right)$, sometime with significant carbon dioxide $\left(\mathrm{CO}_{2}\right)$, heavier hydrocarbon $\left(\mathrm{C}_{2} \mathrm{H}_{6}\right.$, etc.), and/or nitrogen $\left(\mathrm{N}_{2}\right)$. This research covers only $\mathrm{CH}_{4}$ and $\mathrm{CO}_{2}$ only, since these two components are the dominant compositions in research area.

A requirement for CBM wells / reservoir to have the economical gas rate is having enough coal permeability. Permeability is a property of porous media that measures the ability of it to transmit fluids. Most of gas and water flow through the coal cleat system and other fractures. Integrated GRI reservoir studied in San Juan Basin and Black Warrior Basin have produced these following key results: the most important properties to be measured are coal thickness, cleat permeability, gas content, and the sorption isotherm.

Related to the prediction of TKP, several researcher have observed and researched since the 70s. Kim, A.G. (1977), he formulated that methane content was depended on coal rank and pressure. Therefore, the formula was a function of moisture composition, ash composition, and pressure. Mullen, M.J., et.al. (1988) explained new wire-line logging / open-hole logging procedures for CBM and new techniques for evaluating open-hole log in CBM of the Friutland formation in San Juan basin, New Mexico and Colorado. Their formulated gas content was directly as a function of bulk density. Mavor, M.J., et.al. (1997) stated that gas content was a function of ash content in the coal. Bhanja, A.K., et.al. (2008) related the gas content to open-hole log. They formulated that gas content was a function of composite numbers of DT, RHOB, PE, and GR. In 1922, Hawkins, J.M., et.al. developed general correlation for estimating coal adsorption isotherms using open-hole log data. Meanwhile, $\mathrm{Li}$, J., et.al. (2011) formulated the permeability of anthracite coal in the southern Qinshui Basin. The prediction used open-hole $\log$ data. The model noticed that coal permeability can be estimated using the formula of $\mathrm{k}_{\mathrm{f}}=8.50 \times 10^{-4}$ $\mathrm{w}^{2} \phi_{\mathrm{f}}$, in which fracture width (w) from laterolog and fracture porosity $\left(\phi_{\mathrm{f}}\right)$ was from density log.

\subsection{Artificial Intelligence}

In order to predict the TKP, this research uses artificial intelligence methods such as artificial neural network 
(ANN) and adaptive neuro fuzzy inference system (ANFIS).

The ANN type that is used in this research is backpropagation. The word backpropagation is from the method of calculating the weighting gradient changes. Standard backpropagation type changes the weighting gradient using gradient descent algorithm. The variations of standard backpropagation algorithm is in terms of gradient descent using several optimization methods or training rules. Table 1 is several training rules which are used in this research.

Table 1. Backpropagation Training Rules Used in This Research

\begin{tabular}{|c|c|c|c|c|}
\hline No & TRAINING RULE & ALGORITHM & BEST IN & WEAKNESS \\
\hline 1 & Traingdx & $\begin{array}{c}\text { Gradient descent momentum and an } \\
\text { adaptive leaming rate }\end{array}$ & $\begin{array}{l}\text { Useful for some problems. certain situations } \\
\text { in which it is better to converge more slowly } \\
\text { (for example, when using early stopping, may } \\
\text { have inconsistent results if you use an } \\
\text { algorithm that converges too quickly). }\end{array}$ & $\begin{array}{l}\text { Usually much slower than the other } \\
\text { methods, and has about the same } \\
\text { storage requirements as trainrp. }\end{array}$ \\
\hline 2 & Trainlm & Levenberg-Marquardt & $\begin{array}{l}\text { Fastest algorithm on function approximation } \\
\text { problems, particularly for networks that } \\
\text { contain up to a few hundred weights. }\end{array}$ & $\begin{array}{l}\text { As the number of weights in the } \\
\text { network increases, the advantage of } \\
\text { the trainlm decreases. }\end{array}$ \\
\hline 3 & Trainscg & Scaled Conjugate Gradient & $\begin{array}{l}\text { Almost as fast as the trainlm on function } \\
\text { approximation problems (faster for large } \\
\text { networks), and is almost as fast as trainrp on } \\
\text { pattern recognition problems. This algorithms } \\
\text { have relatively modest memory requirements. }\end{array}$ & $\begin{array}{l}\text { Needs more iteration compared to other } \\
\text { Gradient algorithms }\end{array}$ \\
\hline 4 & Trainrp & Resilient Backpropagation & $\begin{array}{c}\text { Fastest algorithm on pattem recognition } \\
\text { problems. The memory requirements for this } \\
\text { algorithm are relatively small. }\end{array}$ & $\begin{array}{l}\text { Does not perform well on function } \\
\text { approximation problems. }\end{array}$ \\
\hline 5 & Trainbr & Bayesian Regularization & $\begin{array}{l}\text { It is desirable to determine the optimal } \\
\text { regularization parameters in an automated } \\
\text { fashion. }\end{array}$ & $\begin{array}{c}\text { For some noisy and small problems this } \\
\text { algorithm can take longer, but often } \\
\text { obtain a better solution }\end{array}$ \\
\hline
\end{tabular}

The generalization techniques used in this research are early stopping (ES) and Bayesian regularization (BR). It verifies network performance by testing different initial conditions. The training process in ES regularization requires at least two groups of datasets, namely training dataset and validation dataset. When the data is limited and the process is function approximation, BR provides better generalization performance than ES. This is because BR does not require validation dataset apart from the training dataset; it uses all the data. In BR technique, network weights and biases are considered as random variables with certain distributions. Regularization parameters are associated with unknown variance associated with the distributions. It is important to train the network until it reaches convergence in this technique.

The neuro-adaptive learning method acts similarly to neural networks. Neuro-adaptive learning techniques provide a method for a fuzzy modeling procedure to learn information about a dataset. ANFIS constructs a fuzzy inference system (FIS) whose membership function parameters are adjusted using either a backpropagation algorithm only or a combination of backpropagation algorithm with least squares method. This adjustment allows the fuzzy systems to learn the dataset in a training (learning) process.

\section{Methods}

The state of art of artificial intelligence (AI) processing are described in this chapter. The flow chart can be seen briefly in figure 2. This research is started from data preparation and processing. It is important to capture all available data to produce applicable final networks for the area as large as possible. Multilayer networks can be trained and result excellent networks which are applicable within the range of dataset. It is important to have the training data spans maximally in the data space because they cannot extrapolate beyond the range of data accurately. The actual data used in this research are large enough in term of coal range distribution. Total numbers of dataset is 315 . The distribution are from lignite (relative young age) to bituminous (relative old age). 


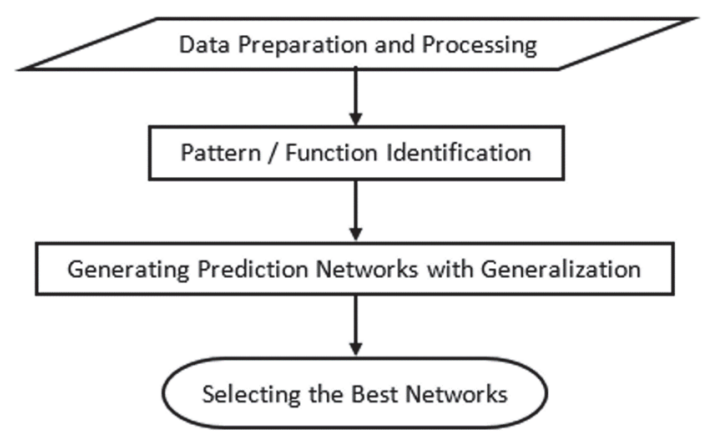

Figure 2. Research Flowchart

The data preparation and processing covers three major processes, they are: data selection, data calibration or normalization, and data grouping. First of all, it is selected seven parameters as the inputs. These parameters are common constituent of open-hole log data. Meanwhile, the output parameters are TKP of CBM reservoir.

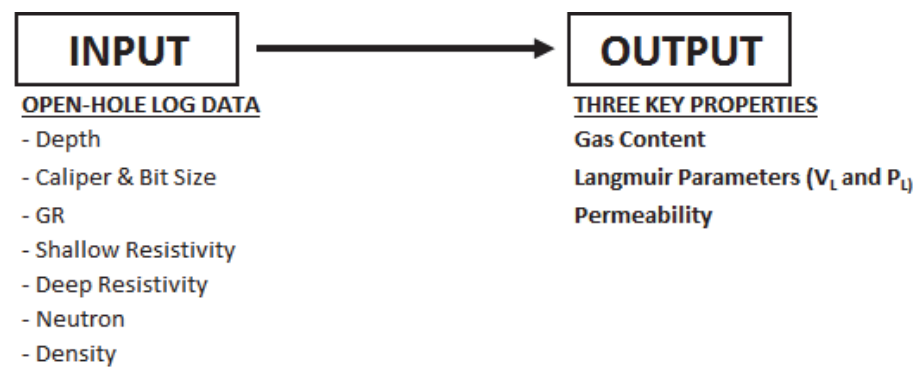

Figure 3. Input-Output in This Research

We can see in figure 3, that all inputs are the constituent of regular open-hole log data, such as: Depth, Caliper, Bit Size, Gamm Ray, Shallow Resistivity, Deep Resistivity, Neutron, and Density. Caliper (C) and bit size (B) will be combined to be one parameter represents the change of well-bore size due to drilling activity. The formula is the different between caliper and bit size divided by bit size or symbolized as (C-B)/B. Total numbers of open-hole $\log$ data used is from 27 wells. Since degree or magnitude of the data are varied, they then are normalized in order to have similar scope within all inputs. Whilst the data which is commonly range in logarithmic scale such as resistivity is converted to be logarithmic form before being normalized.

The normalization and logarithmic formula can be seen in these following equations:

$$
\begin{gathered}
x_{\text {norm }}=x_{\text {MINnorm }}+\frac{\left(x_{\text {MAXnorm }}-x_{\text {MINnorm }}\right)\left(x-x_{\text {MINdata }}\right)}{\left(x_{\text {MAXdata }}-x_{\text {MINdata }}\right)} \\
x_{\log }=\log _{10}(x)
\end{gathered}
$$

Where,

\begin{tabular}{llll}
\hline $\mathrm{X}_{\text {norm }}$ & $=$ Normalized data & $\mathrm{X}_{\text {MINnorm }}$ & $=$ Minimum value of normalized data \\
\hline $\mathrm{x}_{\log }$ & $=$ Logarithmic data & $\mathrm{x}_{\text {MAXnorm }}$ & $=$ Maximum value of normalized data \\
\hline $\mathrm{X}$ & $=$ Actual data & $\mathrm{X}_{\text {MINdata }}$ & $=$ Minimum value of actual data \\
\hline & $\mathrm{X}_{\text {MAXdata }}$ & $=$ Maximum value of actual data
\end{tabular}

All output (TKP) are gas content, Langmuir parameters, and permeability. They are from core analysis and well test results. Basically, those two measurement are the mandatory tasks in developing a CBM reservoir / field. Similar with input data, the distribution of output data are also scatter and varied. But considering the possibility of getting minus results in prediction is exist, then the strategy of converting the data in logarithmic form is the best option in this case.

On the other hand, there is no calibration needed in the input data because of the nature type of the data. The example for calibration or making the data in one datum is production test data. Well-A tested X MMscfd through medium pressure system, meanwhile well-B tested Y MMscfd through lower pressure system. So thus, it 
needs the calibration for data. The way either transforming the well-A data to be well-B's pressure system or vice versa.

One of AI capability is prediction on something which are either as patterns or functions between input-output naturally. The pattern recognition or function approximation are required to ensure the next process 'generating the prediction networks' easier to be achieved. This process is performed using artificial neural network (ANN) and adaptive neuro fuzzy inference system (ANFIS). Both of them are actually the supervised learning algorithms. This research employs Matlab for the whole AI process.

For this pattern or function identification, the ANN selects several training algorithms to be used as shown in table 1 (or table 2). Meanwhile, the ANFIS uses combination of backpropagation and least squared techniques (hybrid) for the training. The process are conducted in a thousand iterations at maximum.

Generating the network for prediction are the main objective of this research. The process basically is almost similar with pattern / function identification process. The difference is advancing the process for generalization to have larger application of the final networks. Two generalization / regularization techniques are used in this research, early stopping and Bayesian regularization. The early stopping technique is standard technique that applied in generating the prediction networks. It is used in both ANN and ANFIS. It is actually the validation or checking process in training process for networks generation. Table 1 (or table 2) shows the algorithms that will be tested to have the best network for prediction. Meanwhile, Bayesian regularization minimizes the combination of squared and weighting factors, and then determines the correct combination to produce a well-generalized network.

The coefficient correlation used in this research is normalized root mean squared error (NRMSE), as shown below:

$$
\begin{aligned}
& R M S E=\sqrt{\left(\frac{\sum_{i=1}^{N}\left(x_{\text {data }, i}-x_{\text {calc }, i}\right)^{2}}{N}\right)} \\
& N R M S E=\frac{R M S E}{\left(x_{M A X d a t a}-x_{M I N d a t a}\right)}
\end{aligned}
$$

Where,

\begin{tabular}{llll}
\hline RMSE & $=$ Root mean square error & $\mathrm{x}_{\text {data, }}$ & $=$ Actual data, $\mathrm{i}$-th \\
\hline $\mathrm{NRMSE}$ & $=$ Normalized RMSE & $\mathrm{x}_{\text {calc., }}$ & $=$ Calculated data, $\mathrm{i}$-th \\
\hline $\mathrm{N}$ & $=$ Total number of dataset & $\mathrm{x}_{\text {MAXdata }}$ & $=$ Maximum value of actual data \\
\hline & & $\mathrm{x}_{\text {MINdata }}$ & $=$ Minimum value of actual data
\end{tabular}

So, the process can be summarized as follows:

1. Prepare the data. Input data from open-hole log data: Depth, (C-B)/B, Gamm Ray, Shallow Resistivity, Deep Resistivity, Neutron, and Density. The output data are from core analysis and well testing results: Gas Content, Langmuir Parameters of $\mathrm{CH}_{4}$ and $\mathrm{CO}_{2}$, and Permeability.

2. Convert the resistivity data to be logarithmic form using equation 3 . Convert all inputs to be normalized (range of -1 to 1 ) using equation 2. Convert all outputs to be logarithmic form using equation 3 .

3. Perform pattern or function identification using the training process of ANN and ANFIS. For the ANN use the sensitivity of all training algorithms in table 1 (or table 2). For ANFIS use the network setting as shown in table 2 .

Table 2. Training Algorithms and Network Information

\begin{tabular}{c|c|c|c} 
NO & METHOD & TRAINING ALGORITHM & NETWORKS \\
\hline \multirow{2}{*}{1} & \multirow{2}{*}{ ANN } & Traingdx, Trainlm, Trainscg, dan Trainrp & $4-16-1$ \\
& & Trainbr & $16-4-1$ \\
\hline 2 & ANFIS & Gradient Descent \& Least Squared & Cluster Radius of 0.35
\end{tabular}

Note. Writing procedure of the ANN method and training algorithms are as follows: 1) ANN_GDX is ANN with Traingdx, 2) ANN_LM is ANN with Trainlm, 3) ANN_SCG is ANN with Trainscg, 4) ANN_RP is ANN with Trainrp, and 5) ANN_BR is ANN with Trainbr. 
4. Plot the errors progression during the pattern or function identification (training process).

5. Generate the networks for prediction using ANN and ANFIS. This process is the complete AI process, started from training, validation / regularization, and testing. Set the number of validation dataset for generalization process in early stopping technique. There is no specific rule to determine the grouping of datasets. But it is controlled by the data distribution and total number datasets, smaller number of total dataset smaller number of validation dataset. In this research, the validation dataset is $4 \%$ of total data (training dataset + validation dataset). The network information is shown in figure 3 .

Tabel 3. Generating Prediction Networks with Generalization

\begin{tabular}{|c|c|c|c|c|}
\hline No & METHOD & TRAINING ALGORITHM & TRAINING : VALIDATION & REGULARIZATION \\
\hline 1 & ANN & $\begin{array}{c}\text { Traingdx, Trainlm, Trainscg, dan Trainrp } \\
\text { Trainbr }\end{array}$ & $96 \%: 4 \%$ & $\begin{array}{l}\text { Early Stopping } \\
\text { Bayesian }\end{array}$ \\
\hline 2 & ANFIS & Gradient Descent \& Least Squared & $96 \%: 4 \%$ & Early Stopping \\
\hline
\end{tabular}

6. Calculate the NRMSE using equation 4 and equation 5.

\section{Results}

This research evaluates the TKP (gas content, Langmuir parameters, and permeability) using available data. The actual data used in this research are large enough in term of coal range distribution. Data distribution are from lignite (relative young age) to bituminous (relative old age). Based on the whole process (refer to figure 2), this research resulted in several parts; pattern identification, generating prediction networks, and selecting the best techniques.

The error plots for pattern/function identification processes from both methods; ANN and ANFIS are shown in figure 4 through figure 7. Meanwhile, the NRMSE for both methods are shown in table 4.

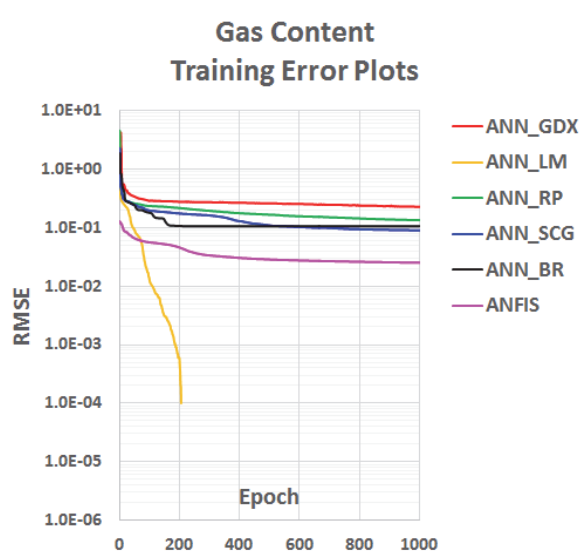

Figure 4. Pattern / Function Identification of Gas Content
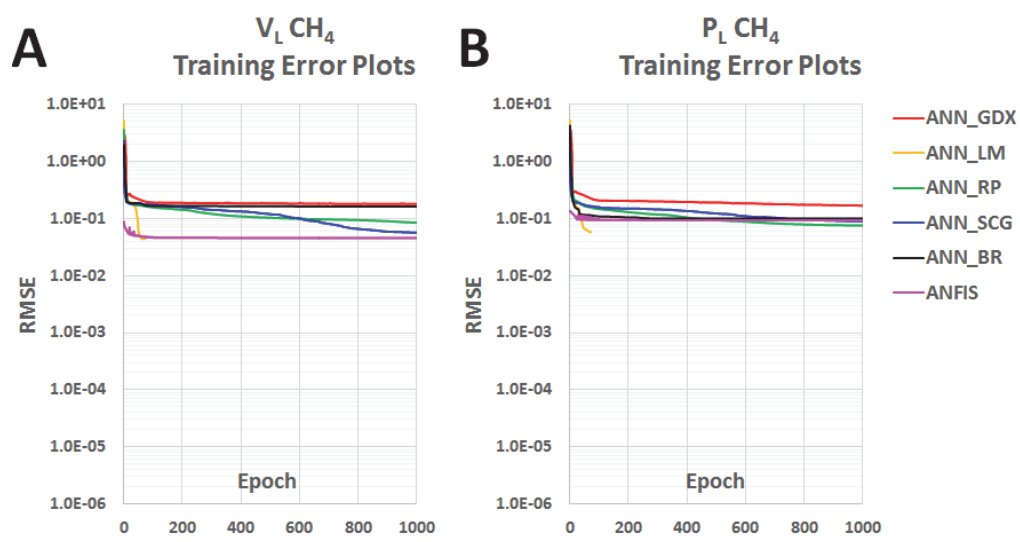

Figure 5. Pattern / Function Identification of Methane's Langmuir Volume (A) and Methane's Langmuir Pressure 

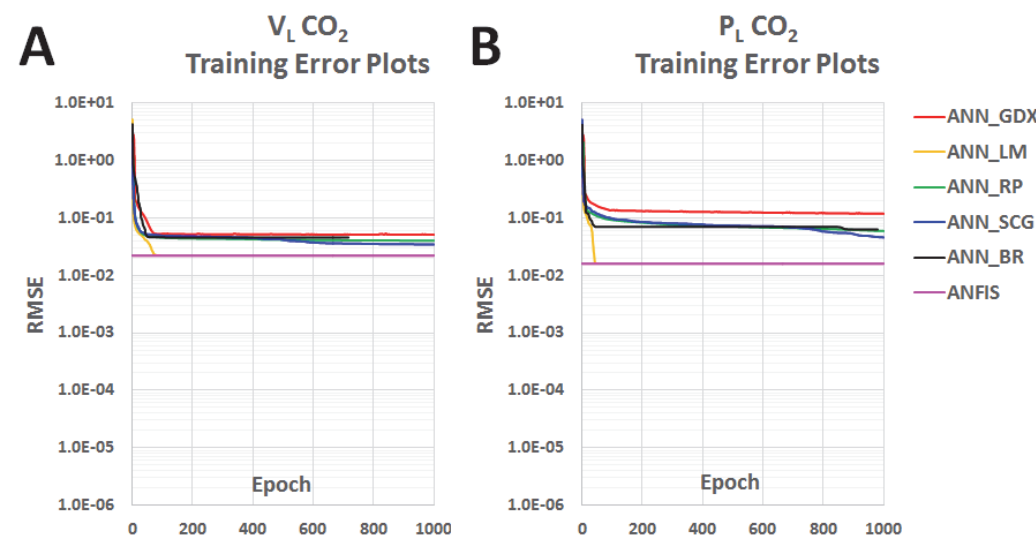

Figure 6. Pattern / Function Identification of Carbon-Dioxide's Langmuir Volume (A) and Carbon-Dioxide's Langmuir Pressure (B)

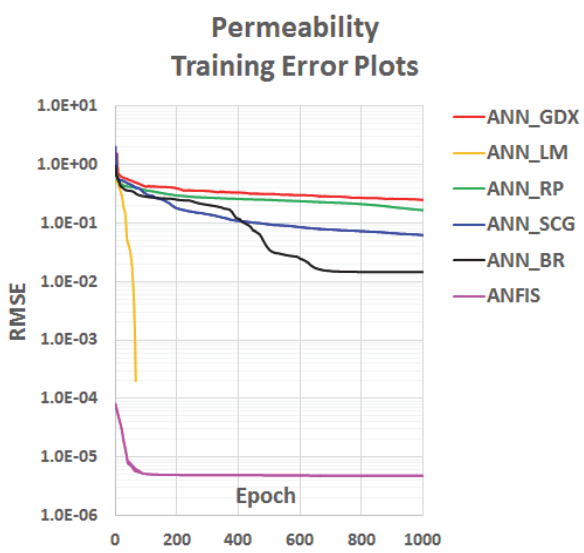

Figure 7. Pattern / Function Identification of Permeability

Then, the process are continued by generating the networks for prediction. The process almost similar to pattern/function identification, but there are additional steps such as generalization/regularization and testing the blind test data.

Table 4. NRMSE of the Network from Pattern/Function Identification

\begin{tabular}{c|c|c|c|c|c|c}
\multirow{2}{*}{ Trining Algorithms } & \multicolumn{5}{|c|}{ ANN } & \multirow{2}{*}{ ANFIS } \\
\cline { 2 - 6 } & traingdx & trainlm & trainrp & trainscg & trainbr & \\
\hline Gas Content & 0.000339 & 0.000000 & 0.000201 & 0.000135 & 0.000159 & 0.000038 \\
\hline Langmuir Parameters: & & & & & & \\
$\mathbf{V}_{\mathbf{L}} \mathbf{C H}_{4}$ & 0.000375 & 0.000092 & 0.000177 & 0.000119 & 0.000340 & 0.000096 \\
$\mathbf{P}_{\mathrm{L}} \mathbf{C H}_{4}$ & 0.000021 & 0.000007 & 0.000009 & 0.000011 & 0.000012 & 0.000011 \\
$\mathbf{V}_{\mathrm{L}} \mathbf{C O}_{2}$ & 0.000109 & 0.000048 & 0.000086 & 0.000074 & 0.000098 & 0.000047 \\
$\mathbf{P}_{\mathrm{L}} \mathbf{C O}_{2}$ & 0.000064 & 0.000009 & 0.000032 & 0.000025 & 0.000034 & 0.000009 \\
\hline Permeability & 0.014299 & 0.000000 & 0.009544 & 0.003560 & 0.000831 & 0.000000
\end{tabular}

\subsection{Gas Content Prediction}

The relationship between open-hole log and gas content is good. It is reflected by the good pattern / function identification and also the prediction. From figure 8, we can see, that ANFIS is the best for gas content prediction with NRMSE of 0.032 . 


\section{Gas Content Prediction}

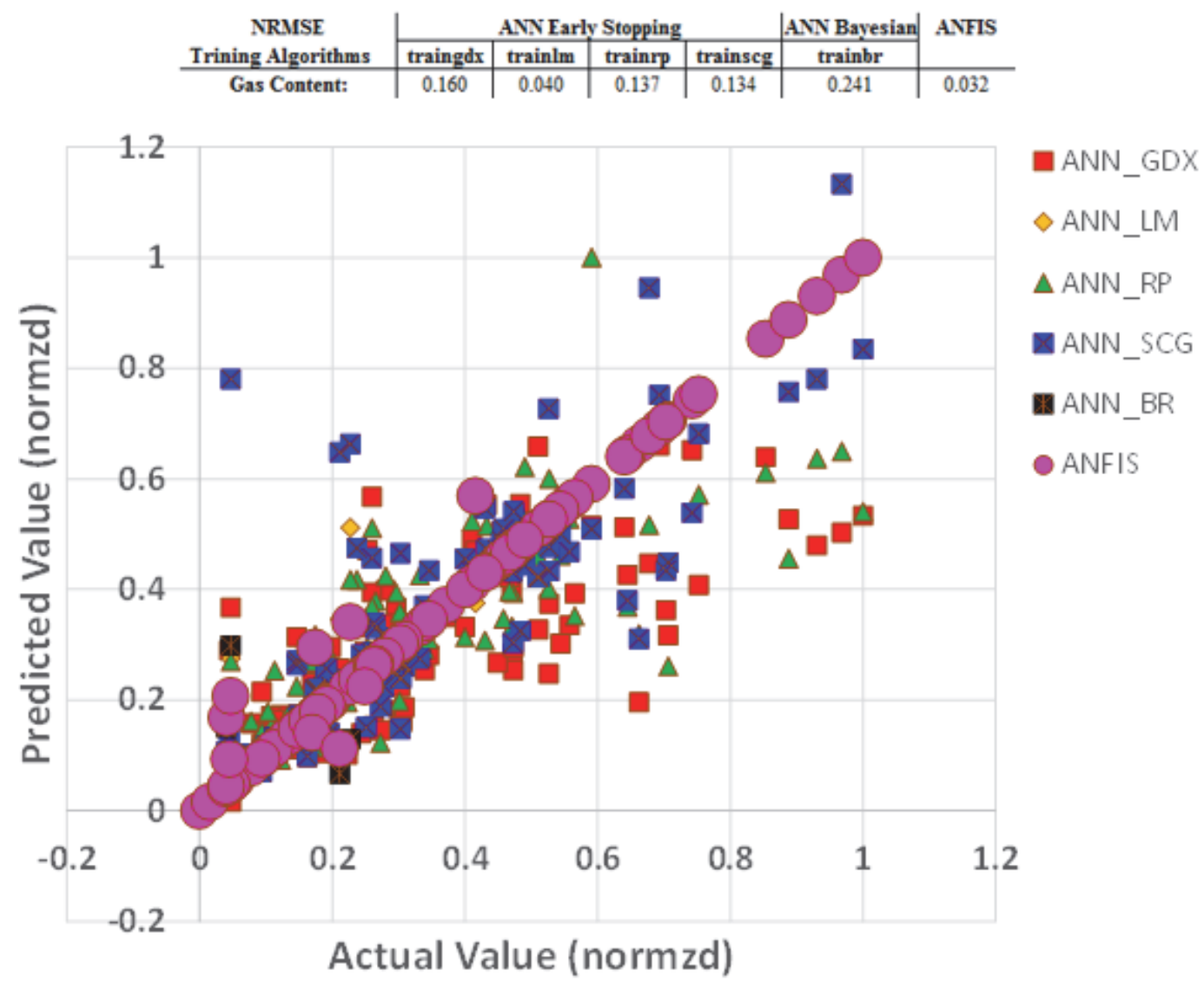

Figure 8. Gas Content Prediction

\section{2 $\mathrm{V}_{\mathrm{L}}$ and $\mathrm{P}_{\mathrm{L}} \mathrm{CH}_{4}$ Prediction}

The relationship between open-hole log and Langmuir parameters of methane is good. It is reflected by the good pattern / function identification and also the prediction. From figure 9, we can see, that ANFIS is the best for $V_{L}$ prediction with NRMSE of 0.106 , and ANN_LM is the best for $P_{L}$ prediction with NMRSE of 0.032 .

A

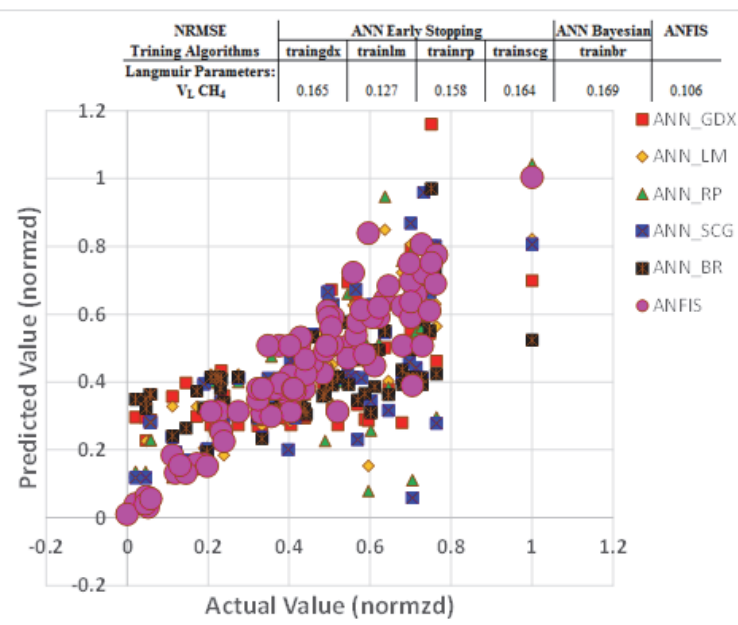

B

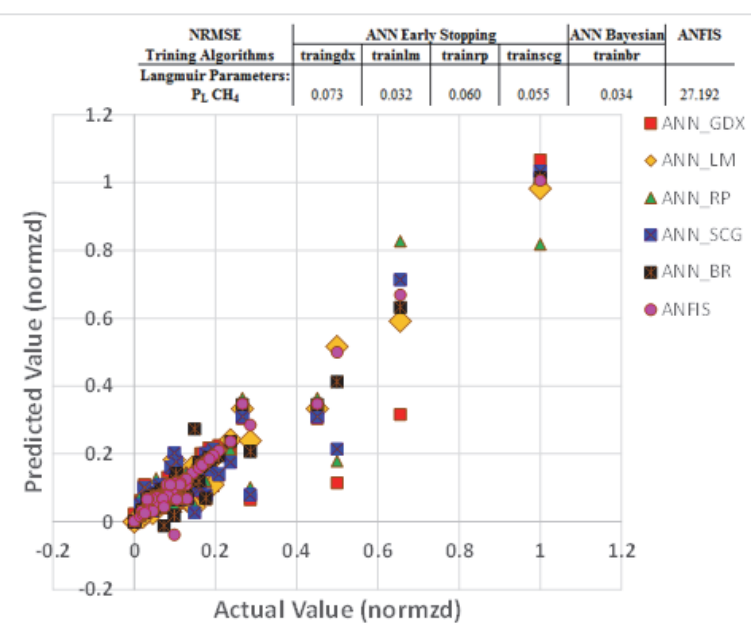

Figure 9. $\mathrm{CH}_{4}$ Langmuir Parameter Prediction 


\section{$3.3 V_{L}$ and $P_{L} \mathrm{CO}_{2}$ Prediction}

The relationship between open-hole log and Langmuir parameters of carbon-dioxide is good. It is reflected by the good pattern / function identification and also the prediction. From figure 10, we can see, that ANN_LM is the best for both $\mathrm{V}_{\mathrm{L}}$ and $\mathrm{P}_{\mathrm{L}}$ prediction with NRMSE of 0.150 and 0.015 respectively.
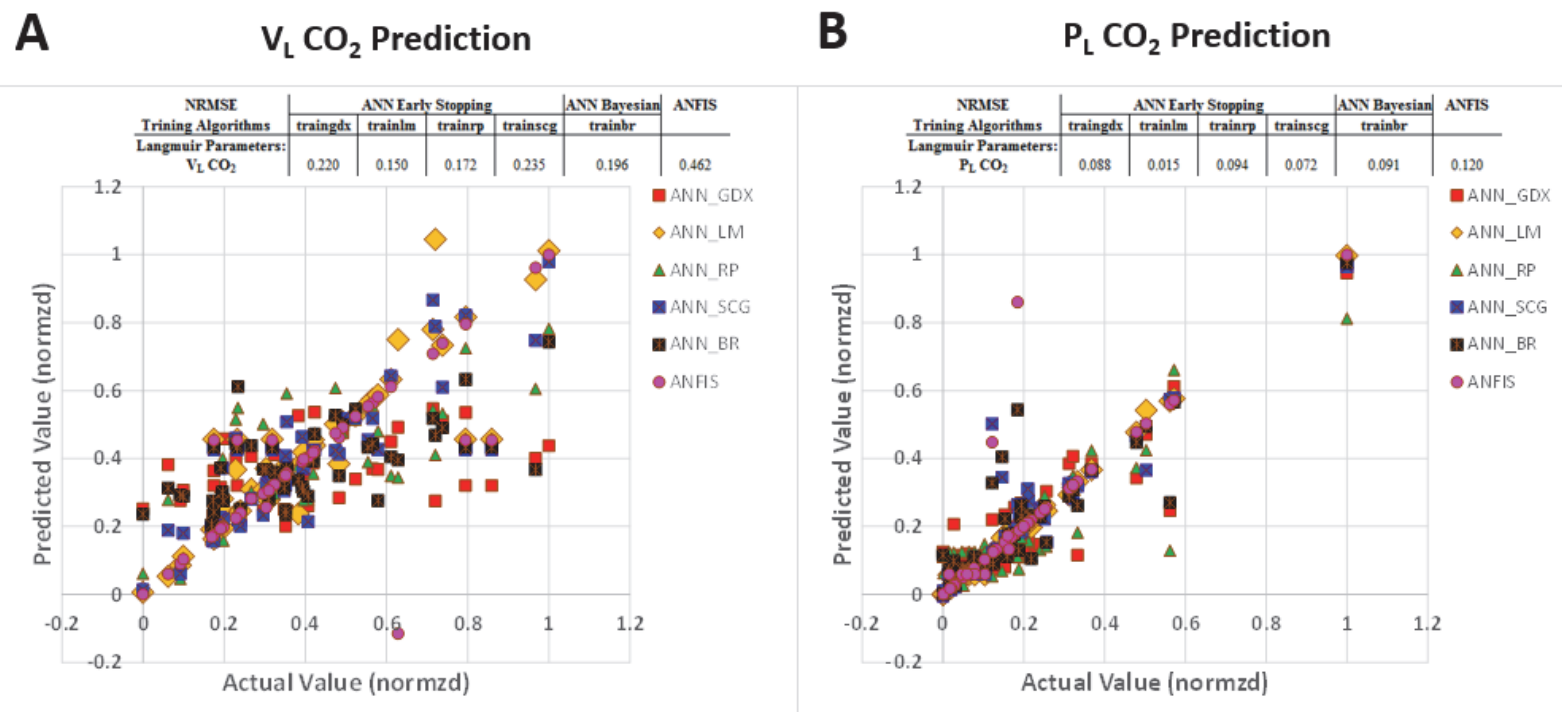

Figure 10. $\mathrm{CO}_{2}$ Langmuir Parameter Prediction

\subsection{Permeability Prediction}

The relation between open-hole log and permeability is good. It is reflected by the good pattern / function identification and also the prediction. From figure 11, we can see, that ANN_BR is the best for this prediction with NRMSE of 0.018 .

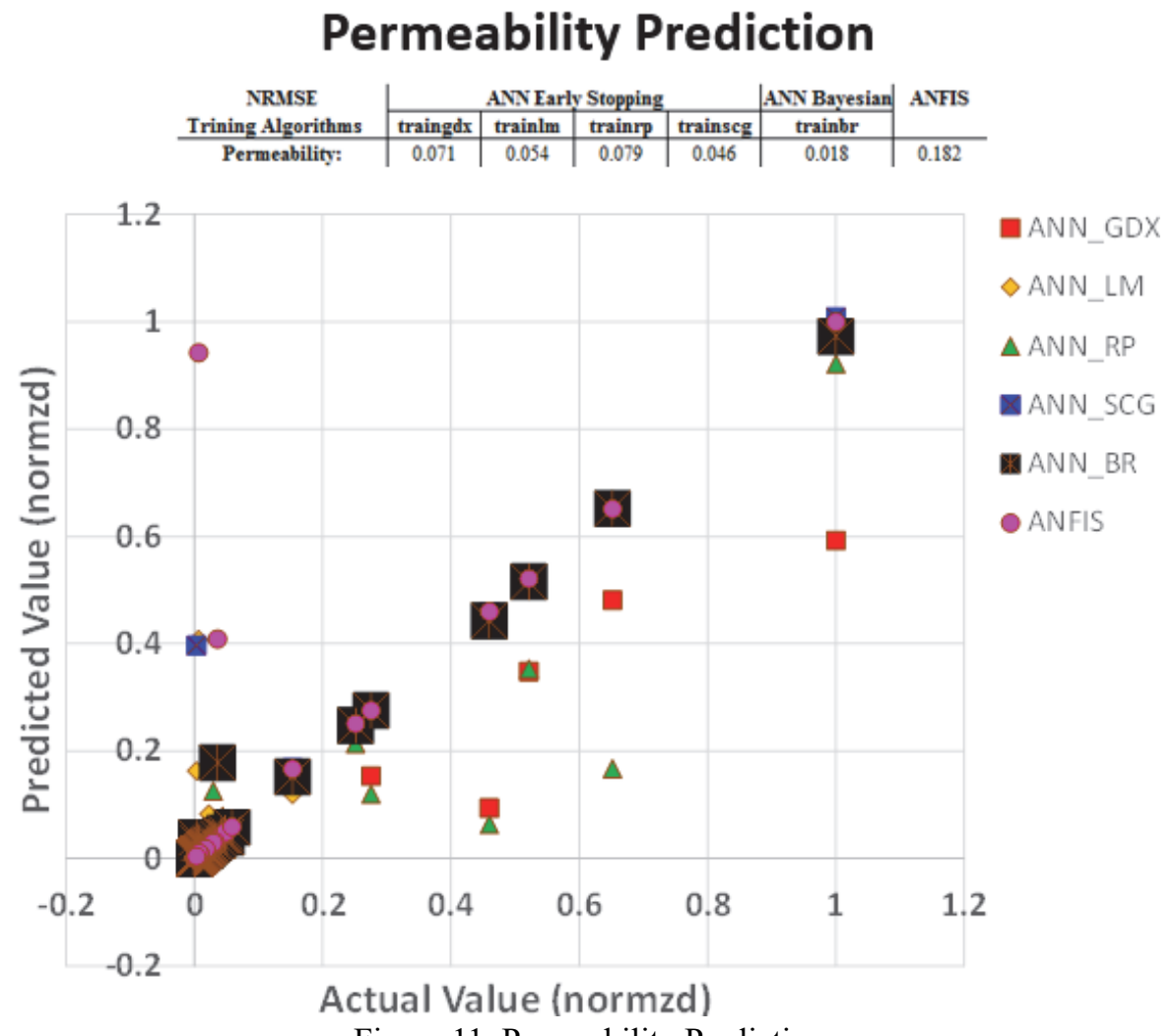

Figure 11. Permeability Prediction 
Table 5. NRMSE of the Network from Prediction Networks Generation

\begin{tabular}{c|c|c|c|c|c|c} 
& \multicolumn{4}{|c|}{ ANN Early Stopping } & ANN Bayesian & \multirow{2}{*}{ ANFIS } \\
\cline { 2 - 6 } Trining Algorithms & traingdx & trainlm & trainrp & trainscg & trainbr & \\
\hline Gas Content: & 0.160 & 0.040 & 0.137 & 0.134 & 0.241 & 0.032 \\
\hline Langmuir Parameters: & & & & & & \\
$\mathbf{V}_{\mathbf{L}} \mathbf{C H}_{4}$ & 0.165 & 0.127 & 0.158 & 0.164 & 0.169 & 0.106 \\
$\mathbf{P}_{\mathbf{L}} \mathbf{C H}_{4}$ & 0.073 & 0.032 & 0.060 & 0.055 & 0.034 & 27.192 \\
$\mathbf{V}_{\mathbf{L}} \mathbf{C O}_{2}$ & 0.220 & 0.150 & 0.172 & 0.235 & 0.196 & 0.462 \\
$\mathbf{P}_{\mathbf{L}} \mathbf{C O}_{2}$ & 0.088 & 0.015 & 0.094 & 0.072 & 0.091 & 0.120 \\
\hline Permeability: & 0.071 & 0.054 & 0.079 & 0.046 & 0.018 & 0.182
\end{tabular}

\section{Discussion}

The pattern recognition or function approximation processes describe us about the relationship between open-hole log and the TKP (gas content, Langmuir parameters, and permeability). From figure 4 through figure 7 and table 4, we can say, generally the ANN and ANFIS can map the relationships between open-hole log and the TKP very well. Several training algorithms in ANN method are excellent to identify the patterns such as ANN_LM and ANN_SCG. Meanwhile, ANFIS method is also excellent for identifying the patterns.

After incorporating the generalization/regularization process into training process and testing the blind test data. As shown in figure 8 through figure 11 and table 5, the prediction process results similar techniques that performed best to predict the TKP. The ANN_LM, ANFIS, and ANN_BR performed best in their specific cases. On the other hand, some of the other techniques are also give small errors in the predictions. Therefore, several techniques can be used for the predictions with certain weighting factor for the best technique.

It is worth to list the best three techniques instead of one for each prediction cases. In this study, the researcher select the best three techniques for each property prediction. So thus, the selected techniques for TKP prediction can be seen in the table 6 .

Table 6. The Best Three Techniques

\begin{tabular}{c|c} 
RESERVOIR PROPERTY & THE BEST THREE TECHNIQUES \\
\hline Gas Content & ANFIS, ANN_LM, ANN_SCG \\
\hline Langmuir Parameters: & \\
$\mathrm{V}_{\mathrm{L}} \mathrm{CH}_{4}$ & ANFIS, ANN_LM, ANN_RP \\
$\mathrm{P}_{\mathrm{L}} \mathrm{CH}_{4}$ & ANN_LM, ANN_BR, ANN_SCG \\
$\mathrm{V}_{\mathrm{L}} \mathrm{CO}_{2}$ & ANN_LM, ANN_RP, ANN_BR \\
$\mathrm{P}_{\mathrm{L}} \mathrm{CO}_{2}$ & ANN_LM, ANN_SCG, ANN_GDX \\
\hline Permeability & ANN_BR, ANN_SCG, ANN_LM
\end{tabular}

The results in table 6 are giving us the optional AI techniques to predict TKP. This will provide us the range of uncertainty for CBM reservoir evaluation.

\section{Conclusion}

From the process of this research, several result can be summarized as follows:

1. Both methods, ANN and ANFIS are able to correlate the relationship between open-hole log and TKP (gas content, Langmuir parameters, and permeability) very well.

2. The techniques which are good to recognize the pattern or to approximate the function between open-hole $\log$ and the TKP are ANN_LM, ANN_SCG, and ANFIS

3. Consistent with point no.2, ANN_LM and ANFIS are also excellent to predict the TKP. 
4. So, the best training algorithms to predict gas content and Langmuir volume of methane are ANFIS, to predict Langmuir pressure of methane and Langmuir parameters of carbon dioxide are ANN_LM, and to predict permeability is ANN_BR.

5. This research also try to capture the uncertainty of reservoir properties and also the range of prediction possibilities by having several techniques (the best three) to predict the TKP as shown in table 6 .

\section{References}

Ahmed, T., \& McKinney, P. D. (2005). Advanced Reservoir Engineering. Unconventional Gas Reservoirs. United States of America. Elsevier.

Bhanja, A. K., \& Srivastava, O. P. (2008). A New Approach to Estimate CBM Gas Content from Well Logs. SPE-115563-MS. https://doi.org/10.2118/115563-MS

Fekete Associates Inc. (2014). Langmuir Isotherm. Retrieved from http://www.fekete.com/SAN/WebHelp/FeketeHarmony/Harmony_WebHelp/Content/HTML_Files/Referen ce_Material/General_Concepts/Langmuir_Isotherm.htm

Hawkins, J. M., Schraufnagel, R. A., \& dan Olszewski, A. J. (1992). Estimating Coalbed Gas Content and Sorption Isotherm Using Well Log Data. SPE-24905-MS. https://doi.org/10.2118/24905-MS

Kim, A. G. (1977). Estimating Methane Content of Bituminous Coalbeds from Adsorption Data. United States Department of the Interior.

Li, J., Liu, D., Yao, Y., Cai, Y., \& Qiu, Y. (2011). Evaluation of the Reservoir Permeability of Anthracite Coals by Geophysical Logging Data. China. International Journal of Coal Geology. https://doi.org/10.1016/j.coal.2011.06.001

Mavor, M. J., \& Nelson, C. R. (1997). Coalbed Reservoir Gas-in-Place Analysis. Gas Research Institute. Chicago. Illinois.

Mavor, M. J., Paul, G. W., Saulsberry, J. L., Schafer, P. S., Schraufnagel, R. A., Steidl, P. F., Sparks, D. P., \& Zuber, M. D. (1996). A Guide to Coalbed Methane Reservoir Engineering. Gas Research Institute. Chicago. Illinois.

McLennan, J. D., Schafer, P. S., \& Pratt, T. J. (1995). A Guide to Determining Coalbed Gas Content. Gas Research Institute. Chicago. Illinois.

Mullen, M. J. (1989). Coalbed Methane Resource Evaluation from Wireline Logs in the Northeastern San Juan Basin: A Case Study. SPE-18946-MS. https://doi.org/10.2118/18946-MS

Rachmat, S., \& Hadad, A. F. (2014). Pattern Recognition to Predict Langmuir Parameters in Coalbed Methane Using Neural Network. IATMI. Jakarta

The MathWork, Inc. (1992-2014). Beale, M. H., Hagan, M. T., \& Demuth, H. B. (2014). Neural Network Toolbox ${ }^{\mathrm{TM}}$ User's Guide. MATLAB ${ }^{\circledR}$. The MathWork, Inc.

The MathWork, Inc. (1995-2014). Fuzzy Logic Toolbox ${ }^{\mathrm{TM}}$ User's Guide. MATLAB®. The MathWork, Inc.

\section{Copyrights}

Copyright for this article is retained by the author(s), with first publication rights granted to the journal.

This is an open-access article distributed under the terms and conditions of the Creative Commons Attribution license (http://creativecommons.org/licenses/by/4.0/). 